Document downloaded from:

http://hdl.handle.net/10251/92203

This paper must be cited as:

Díaz Marín, MDC.; Aura Castro, E.; Sánchez Belenguer, C.; Vendrell Vidal, E. (2016). Cyclododecane as opcifier for digitalization of archaeological glass. Journal of Cultural Heritage. 17:131-140. doi:10.1016/j.culher.2015.06.003

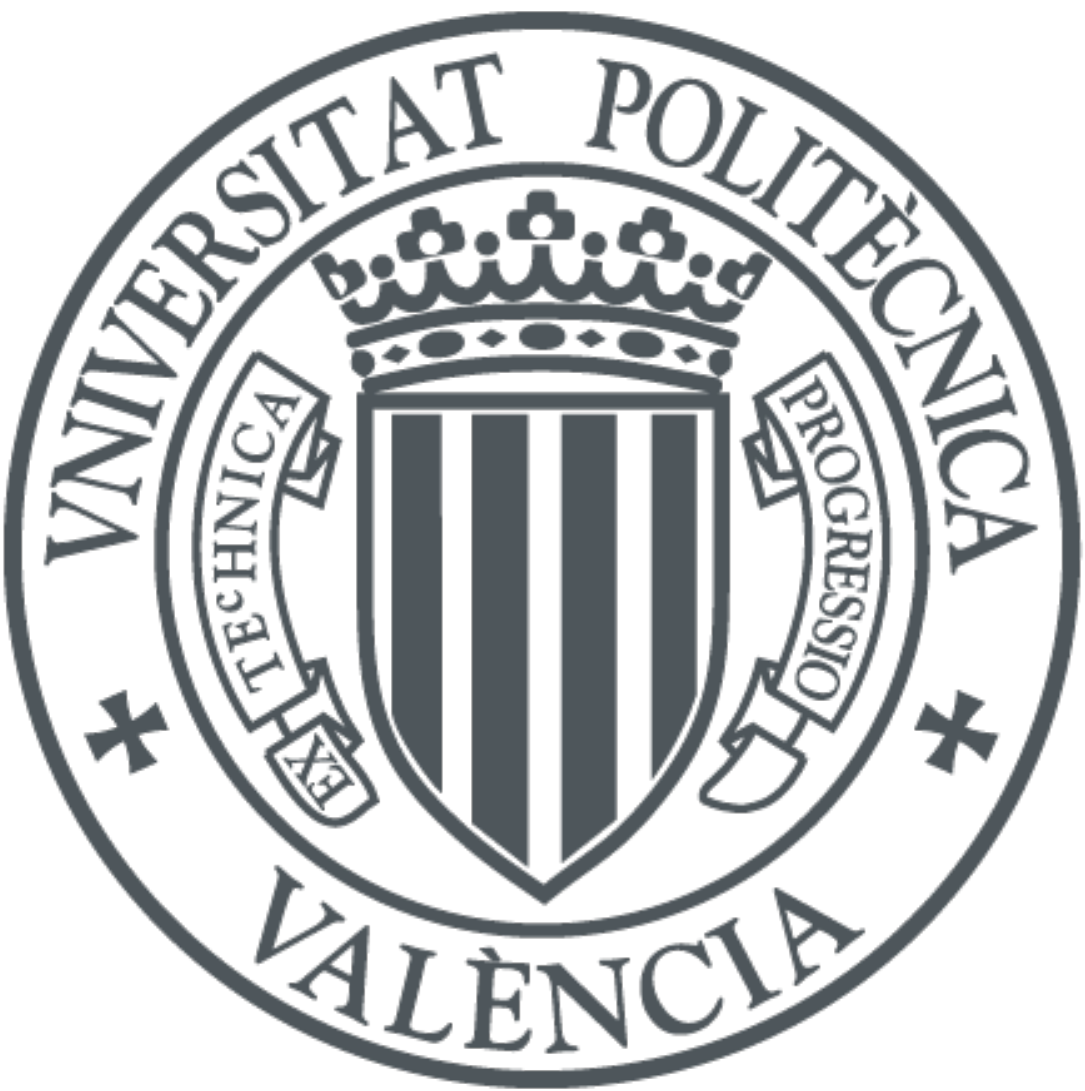

The final publication is available at

https://doi.org/10.1016/j.culher.2015.06.003

Copyright Elsevier

Additional Information 


\title{
Cyclododecane as Opacifier for Digitalization of Archaeological Glass
}

\author{
M. C. Díaz ${ }^{\mathrm{a}}$, E. Aura ${ }^{\mathrm{a}}$, C. Sánchez ${ }^{\mathrm{b}, \mathrm{c}, *}$, E. Vendrell ${ }^{\mathrm{b}}$ \\ \{madiama, eaura\}@crbc.upv.es, \{carsanb1, evendrell\}@ai2.upv.es \\ ${ }^{\text {a }}$ Instituto U. de Restauración del Patrimonio. \\ Universitat Politècnica de València. Valencia, Spain \\ ${ }^{\mathrm{b}}$ Instituto U. Automática e Informática Industrial. \\ Universitat Politècnica de València. Valencia, Spain \\ ${ }^{\mathrm{c}}$ Institute for Transuranium Elements. European Commission \\ Joint Research Centre (JRC). Ispra (VA), Italy
}

\begin{abstract}
This paper faces the problem of acquiring archaeological artifacts using triangulation based 3D laser scanners and focusing on reflective/refractive surfaces. This kind of artifacts are mostly made of glass or polished metal, and the properties of their surfaces violate most of the fundamental assumptions made by vision algorithms. Also, the unique and fragile nature of archaeological artifacts adds an extra limitation to the acquisition process: using industrial whitening sprays has to be avoided, due to the physicochemical processes required to clean the surface after scanning and because the chemical properties of these sprays may damage the original object. As an alternative to them, a new application of a common conservation material is proposed: the use of cyclododecane as a whitening spray. Thanks to its chemical stability and to the fact that it sublimes at room temperature, together with its good film-forming capabilities, a set of evaluation tests is presented to prove that the error introduced by the opaque thin layer created on the surface of the artifact is smaller than the accuracy of the $3 D$ scanner and, thus, no acquisition errors are introduced. A comparison with general-purpose industrial whitening sprays is also presented, and achieved results show no significant differences in the quality of the resulting $3 D$ models.
\end{abstract}

Keywords: cyclododecane / 3D acquisition / archaeological glass / opacifier / whitening spray

\section{RESEARCH AIMS}

This paper proposes the use of cyclododecane, a very common conservation material, for 3D acquisition of reflective/refractive archaeological artifacts. Thanks to the fact that it sublimes at room temperature leaving no residuals and its good film-forming capabilities, its use as an opacifier allows using general-purpose triangulation based 3D laser scanners without damaging original objects. A set of evaluation experiments is introduced in order to demonstrate that quality of achieved results is similar to general-purpose industrial whitening sprays.

\section{INTRODUCTION}

Acquiring 3D models of archaeological artifacts allows having detailed digital representations of them that can be used for analytical or dissemination purposes. Furthermore, operating on digital models prevents damaging original artifacts and facilitates the access to the research community. 
In order to create these digital representations, 3D acquisition devices are used to capture the topology of real objects. There are several technologies to perform such operation, like contact scanners based on physical touch, photogrammetry approaches, time of flight cameras and triangulation laser scanners, amongst others. Last ones are the most common in heritage applications, given the balance between accuracy and cost that they offer.

One of the main problems of triangulation laser scanners is that they require opaque surfaces in order to apply the vision algorithms that allow capturing the geometry of real objects. This is not an important restriction when working with stone or clay artifacts, but becomes a big drawback when working with archaeological glass: reflective/refractive surfaces violate most of the fundamental assumptions made by vision algorithms. For instance, they cause the projection of a background scene to the image plane to be deformed. Furthermore, this projection can vary from one viewpoint to the next and the reflection of light by the surface complicates the reconstruction process.

To address this problem, industry solutions commonly use whitening sprays designed to create an opaque thin film over the object's surface. This way, acquisition using triangulation laser scanners can be performed and, if the film is thin enough, measurement errors introduced by the sprays can be ignored (since 3D scanners accuracy is orders of magnitude coarser).

The main problem with these sprays is that they are not suitable for archaeological fragments: after the acquisition has been performed, removing the spray layer requires lot of rubbing and/or the use of strong solvents. Given the unique nature of the fragments and their fragility, neither of these two procedures can be applied because their surface might be damaged. Also, chemical stability is a common requirement for manipulating these kind of artifacts and none of the available commercial spray satisfies this condition.

To provide a solution to the acquisition problem in the field of archaeology, a new application of a common conservation material is proposed: the use of cyclododecane as whitening spray. Cyclododecane (abbreviated as CDD) is a volatile cyclic alkaline $\mathrm{C}_{12} \mathrm{H}_{24}$ that is solid at room temperature. For being non-polar and compound exclusively by carbon and hydrogen, it is an inert material whose most attractive characteristic is that it sublimes, eliminating additional chemical or physical treatment steps to remove it. Its physicochemical properties include good film-forming capabilities, insolubility in water, solubility in organic solvents and low toxicity, which render the compound particularly useful in the field of cultural heritage. In fact, in the latest years it has become very common in this field as a temporary consolidant, sealant and hydrophobic protecting coating for fragile materials (paintings, ceramics, papers, textiles...).

Cyclododecane can be applied in different manners but, for the proposed use, spray seems the most convenient since it is the one that creates the thinner film. After applying it to an object, it can be observed that its surface turns opaque and white, which are the most desirable conditions for laser scanning purposes. Immediately after the application, the thin layer starts to sublimate (pass from solid to gas state).

For CCD to be useful in acquisition it has to be proven that, after applying it, the fragment can be correctly acquired and that the error introduced by the thin layer created is smaller than the accuracy of the 3D scanner used. This paper presents a set of evaluation tests that have been designed to demonstrate that CCD does not interfere in the acquisition stage and to compare it with some common industrial whitening acquisition sprays.

\subsection{Previous Works}

3D scanning technology has been widely used in cultural heritage applications for inspection, documentation and research purposes. However, the presence of glass artifacts in this kind of 
applications is scarce: a study focused on the acquisition of historical stained glass windows can be mentioned [1], together with another one focused on old photographic plates [2]. In both cases, glass was painted or covered by an emulsion, which allowed performing the digitalization by making the original artifacts opaque. When considering archaeological glass, 3D acquisition is even more challenging: objects are normally found very fragmented and are extremely fragile. This makes their study very difficult, since handling them normally is accompanied by a complex restoration process.

Nowadays, 3D acquisition of archaeological glass is an unresolved problem. However, in the last years, lot of effort has been put into the development of general-purpose 3D acquisition systems focused on specular and transparent objects. A complete and exhaustive study on the state-of-the-art of these techniques can be found in [3-4], where a set of solutions are introduced that allow acquiring transparent, semi-transparent and highly reflective objects. Some of the proposed solutions consider the fabrication of specific devices for the acquisition of transparent glass using structured UV light [5], infra-red cameras [6], polarization and phase-shifting devices for the acquisition of translucent artifacts [7] or even submerging the original artifact inside a tank filled with a fluorescent fluid [8]. Other solutions combine the use of a video camera recorder, a moving spotlight and a small sphere [9] or a turntable together with a set of cameras, lights and monitors [10]. All these systems are potentially useful, but with a main drawback: they are focused to specific objects and designed to solve special problems, so they cannot be applied to archaeological glass because of their strict restrictions.

An efficient alternative that does not require modifying current general-purpose capturing devices is the use of an anti-reflective spray for transparent or brilliant surfaces, in order to make the opaque and matte. This solution is very versatile and cheap, so its use is very common in industrial applications to face the acquisition of complex reflective/refractive objects. However, general-purpose sprays cannot be used with archaeological glass, given that removing the spray may damage the original surface. To face this problem, the use of cyclododecane as opacifier is proposed in this paper.

CCD started to be used in conservation and cultural heritage applications in the mid 90's and was originally proposed by Hans Michael Hangleiter, Elisabeth Jägers and Erthard Jägers [11] when looking for an easy-to-remove substance that could be applied as a consolidant and temporal protector. Since then, it has been used for many different materials like stone, ceramics, paper, fossils and glass amongst others [12-15]. The most interesting property of CCD for curators of cultural heritage is the fact that it sublimates at room temperature, leaving no residuals. This feature explains such an extended use as temporary consolidant, protective coating, barrier layer and adhesive [16-17]. As far as we can tell, there is no previous research on applying this substance for 3D acquisition purposes.

A similar product to CCD are developer sprays and an example of their use in cultural heritage applications can be found in [18]. In this paper, authors evaluate four different scanners and, for one of the targeted objects, they apply this spray to facilitate acquisition and registration of the resulting point clouds. This kind of sprays are not real paintings but specifically formulated products created to perform color penetration tests in the detection of fissures and cracks of different surfaces. Developers allow the inspection and detection of a dye that has been previously introduced inside the cracks to characterize. They form extremely thin films and, at the same time, they provide a homogeneous coverage. In most of the cases, the choice of the proper developer depends on the geometry of the surfaces, the relief and the number of inspections to make. Nowadays, their application field has been extended, being commonly used for 3D acquisition of shiny, translucent or very dark objects. However, the use of these products is not suitable for cultural heritage applications [19] because they may alter the surface of artifacts. 
Anselmi et al. [20] studied the behavior of CDD applied as spray over non-porous surfaces by using NMR profilometry and FTIR reflectance spectroscopies. From their results, it is interesting to consider that CDD does not penetrate and remains in the surface layers, consistent with the level of abrasion of the surface tested. Stein et al [21] applied melted cyclododecane and saturated solution on glass slides and other materials. Sublimation was monitored in three ways: visually, as function of weight loss over time and by GC-MS. Authors also here found that CDD sublimed without residues. However, in others studies with microscopy, FTIR and GC-MS it had been found that test applications of cyclododecane on glass microscope slides show that some material, possibly an impurity, remains after sublimation [22]. According to these studies, it is recommended to use high quality formulations of this product, to reduce the amount of residuals.

Walters [23] look at sublimation rates by monitoring for weight loss of melted CCD films. Results show long sublimation periods, basically because the product was applied in a melted resin form. When a spray solution is used, the results are quite different: the layer is softer and thinner, providing low resistance to pressure. The spray has the resin dissolved in an extremely volatile dissolvent, a mix of methane and butane, which also acts as the propellant. This mix decreases the evaporation time in comparison to the solid resin.

M. Neurne and M.O. Hubert applied CCD for consolidation and temporal protection of glass [24] and, according to their results, in order to get a similar result as developer films, application distance of CCD as spray has to be around $10 \mathrm{~cm}$.

\subsection{Document Organization}

Next section covers the previous related works in the field of 3D acquisition of reflective/refractive objects and other different applications of cyclododecane in the field of cultural heritage. Then, in Section 3, the results of a set of evaluation tests are presented to measure the sublimation speed of $\mathrm{CCD}$, the error introduced during the 3D scanning and its particle size. Section 4 shows some acquisition results using cyclododecane and, finally, Section 5 presents the conclusions.

\section{EXPERIMENTAL}

In order to evaluate how CCD performs as a whitening spray, to measure the error introduced in the acquisition process and to compare it with other general-purpose industrial whitening sprays, a set of evaluation experiments have been developed.

First experiment deals with the sublimation speed of CCD in accelerated conditions (by directing a hairdryer to an over-sprayed object) and shows the most interesting characteristic of this material. Second and third experiments deal with the measurement of the error introduced in the acquisition process when using CCD, and compares this error with other three common whitening sprays: Helling Developer U 89 (referred as U89 in the tests), Helling Developer D 70 (referred as D70 in the tests) and OPN Developer White (referred as WHITE in the tests). Fourth experiment shows the particle size of each spray using an electronic microscope and estimates the error introduced by each one of them, independently of the 3D scanner used. Finally, fifth experiment estimates the sublimation speed of CCD in order to establish the time window in which the spray can be used as opacifier for acquisition purposes.

For second, third and fifth experiments, the scanner used is a Konica Minolta VIVID 900 with a $24 \mathrm{~mm}$ lenses that provides an average accuracy of $600 \mu \mathrm{m}$, according to the manufacturer specifications. 


\subsection{Sublimation}

According to [11], the average sublimation speed of CCD under normal conditions has been observed to be of $0.03 \mathrm{~mm}$ each 24 hours but there are additional factors that affect this behavior: film thickness and density, substrate porosity, atmospheric temperature and pressure and air exchange over the surface of the film. This way sublimation can be accelerated by directing a hair dryer over the surface of the fragment, or retarded by reducing the airflow over the surface of the object (sealing it, for example).

Fig. 1 shows the accelerated sublimation process (by pointing a hair dryer towards the object, at $50^{\circ}$ Celsius), where the artifact has been over-sprayed to better illustrate this concept. The complete video sequence can be found together with this paper.
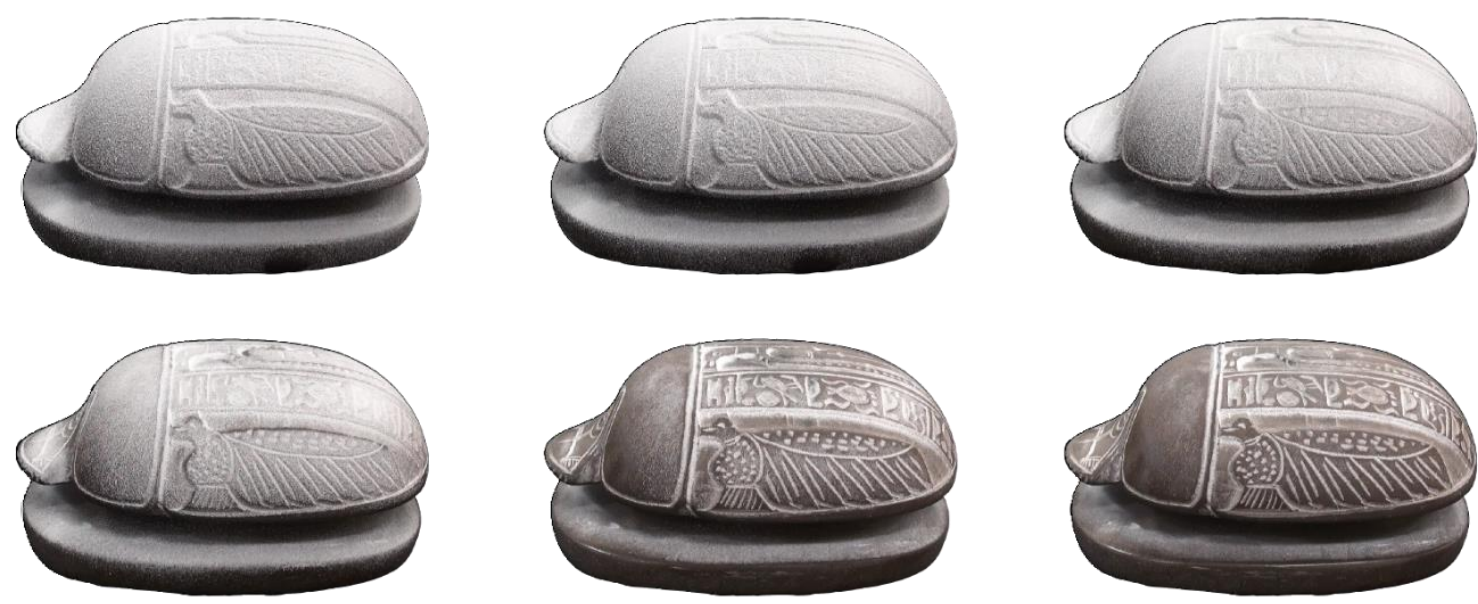

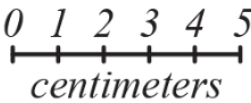

Figure 1: From left to right and top to bottom, the sublimation of cyclododecane applied on an artifact. Images are captured in 45 seconds intervals since the application of the spray (top-left picture). The process has been accelerated pointing a hair dryer towards the object. It is important to remark that, the white areas on the last picture are from the original artifact, since cyclododecane has completely sublimated.

\subsection{Calibration Chart}

This experiment consists on scanning a flat calibration chart and comparing the acquired point cloud with the optimal plane that better fits it. Residuals are expressed as signed distances between this plane and each point, and displayed together in a histogram. Average errors and standard deviations are calculated together with the two $2.5 \%$ intervals containing the worst samples (one for positive distances and another for negative ones).

The proposed experiment has been performed without applying any whitening spray (so the error distribution of the sensor can be characterized), with the 3 commercial whitening sprays commented before and with cyclododecane spray. For this experiment and the next ones, a colorimeter has been used in order to have fair comparisons: spray is applied on the surface, until a specific white tone is achieved.

Results of this experiment, shown in Fig. 2, reveal no significant differences between using or not using any spray, and between cyclododecane and the other commercial solutions. As it can 
be appreciated, $95 \%$ of the samples are always in a range almost centered in 0 , and with $600 \mu \mathrm{m}$ of size, which makes sense given the scanner's accuracy.
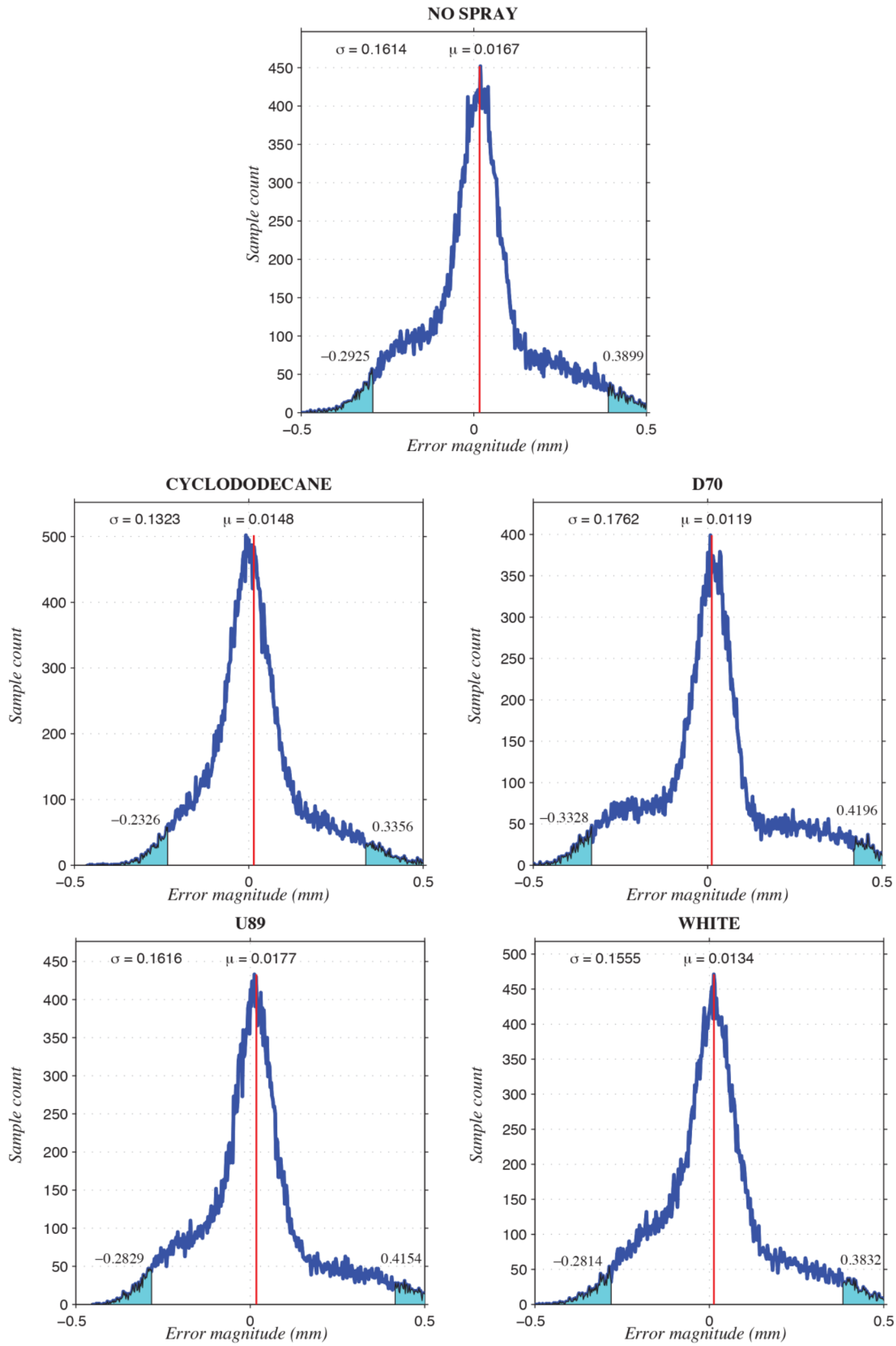

Figure 2: Residuals for the calibration chart after applying each spray, with respect to the optimal plane. The red line indicates the signed average error. Blue intervals on both sides represent (each one) a $2.5 \%$ of the total acquired samples. 


\subsection{Irregular Objects}

This experiment uses more complex shapes to evaluate each spray's performance in a more realistic case. First object has a smooth surface with soft curvatures (like a typical glass artifact), and second has strong incisions.

Given that, in this case, no optimal surface can be approximated, residuals are calculated in a different way: starting from an opaque object, a ground-truth model is acquired. Without moving the object and leaving the scanner focus fixed, the spray is applied until the desired level of white is achieved. Then, the model is acquired again, and both point-clouds are registered (because, even not focusing the scanner again, a constant shift between acquisitions has been observed). Residuals are expressed as point-to-point distances between closest neighbors in both point clouds.

To prevent measurement errors, each object has been scanned three times with each spray, and no object has been re-used after each test. This way, 12 identical replicas of each object have been used: after the acquisition with one spray, the object is discarded for further tests, so residuals remaining in the surface do not alter next measurements.

Figures 3 and 4 show the achieved results expressed as the total number of samples of the three acquisitions performed for each spray. 

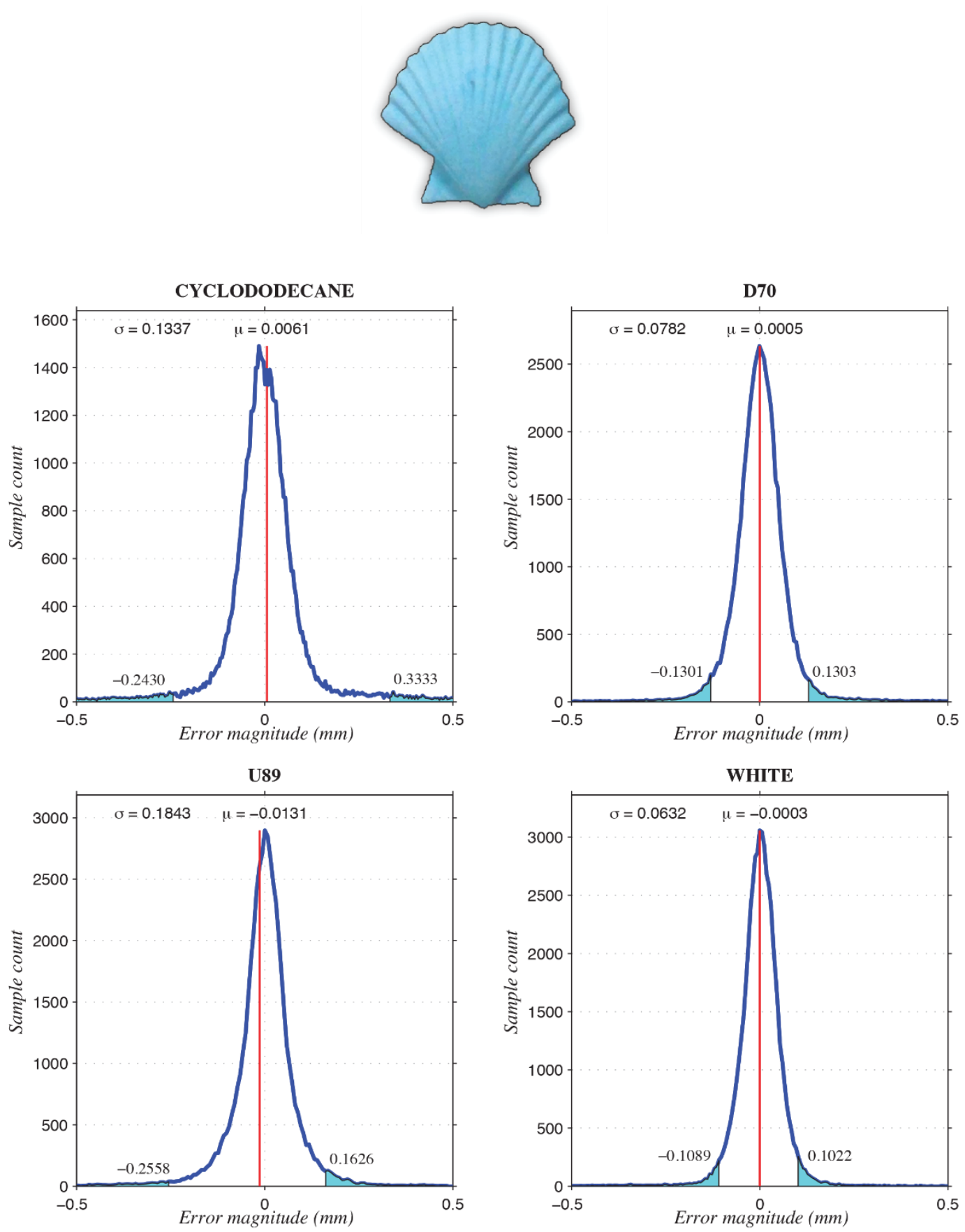

Figure 3: Residuals for model CO after applying each spray, with respect to the same model/pose before applying the spray, and properly registered. The red line indicates the signed average error. Blue intervals on both sides represent (each one) a $2.5 \%$ of the total acquired samples. This model presents an irregular shape with some smooth curvature on the surface. Results show that cyclododecane behaves slightly worst than the alternatives, but always inside the scanner's accuracy range. 

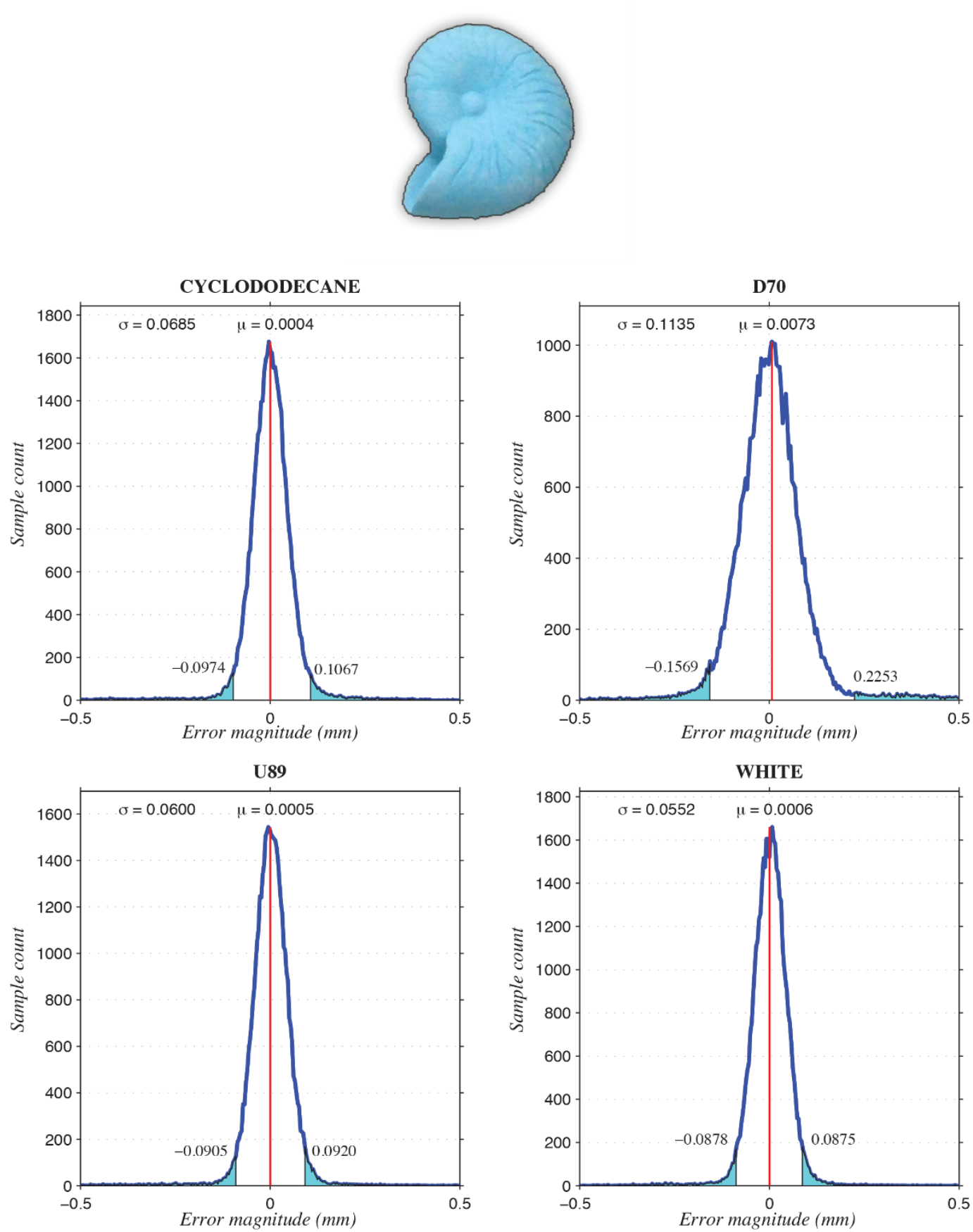

Figure 4: Residuals for model MO after applying each spray, with respect to the same model/pose before applying the spray, and properly registered. The red line indicates the signed average error. Blue intervals on both sides represent (each one) a $2.5 \%$ of the total acquired samples. This model presents an irregular shape with strong incisions on the surface. As happened with previous objects, all four sprays present an error distribution very similar, and inside the scanner's accuracy range and, in this case, CCD performs better than D70. 


\subsection{Electronic Microscope}

As previous results suggest, all four compared products are suitable for acquiring 3D models of archaeological fragments. However, a simple visual inspection of the surface of the fragment after applying the spray reveals differences between cyclododecane and the other products: the white film created by cyclododecane shows more irregularities than the other sprays, suggesting a bigger particle size.

To confirm this, and measure the differences, a set of pictures has been taken using an electronic microscope. In Fig. 5 it can be appreciated how cyclododecane particles are considerably bigger than the other products. However, a closer look to the 5.000 augments picture of cyclododecane (top-right image) clearly shows that the particle size is around $10 \mu \mathrm{m}$, which is one order of magnitude smaller than current laser triangulation scanners' accuracy. This observation confirms previous experiment results, and explains the empirical visual differences between cyclododecane and the other sprays.

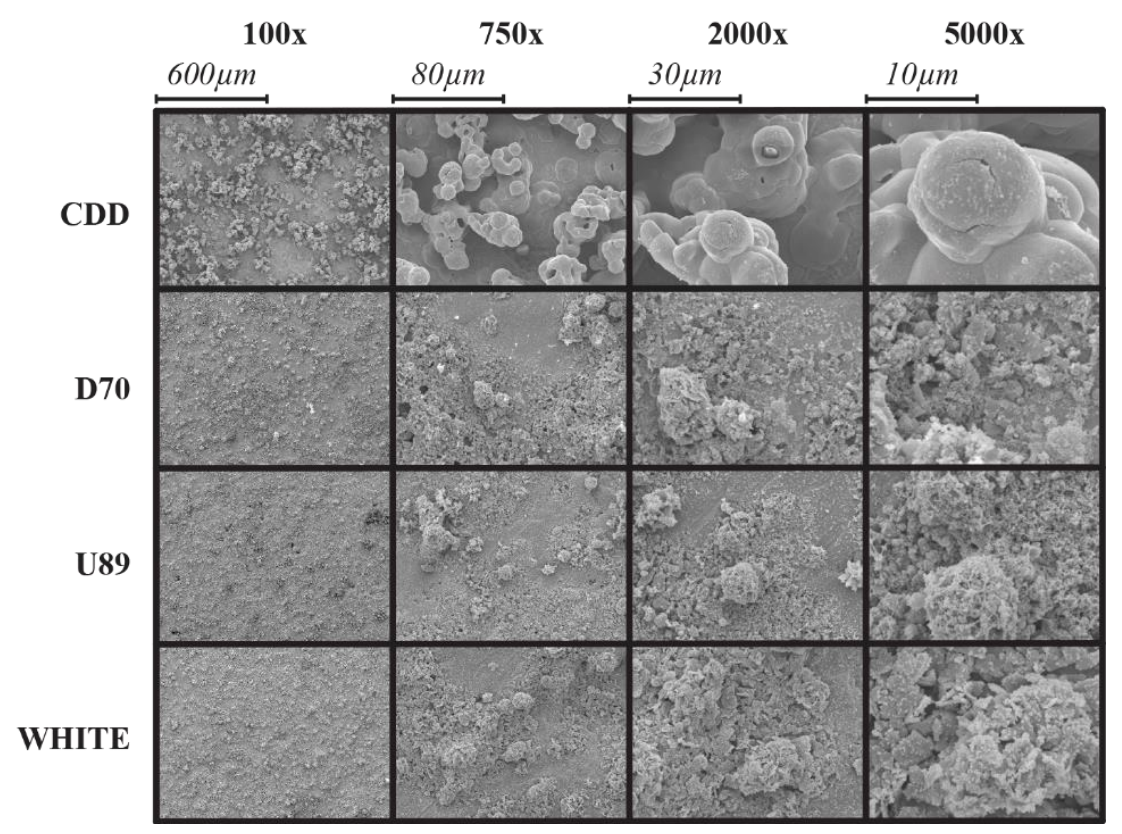

Figure 5: Electronic microscope comparison of whitening sprays at different scales. Notice how, even having a bigger particle size than the other sprays, cyclododecane is still small enough to not interfere with laser scanners.

\subsection{Sublimation speed}

In order to estimate the effective time in which CCD can be used as opacifier for acquisition purposes, two objects have been selected: a big flat glass with some roughness on its surface and a glass pot with more pronounced reliefs and irregularities. Both objects have been sprayed and scanned several times in a fixed pose.

Taking as reference model the point cloud acquired immediately after applying the spray, subsequent acquisitions have been compared against it, focusing on two different estimators for the sublimation speed: the number of valid measures returned by the scanner and the average deviation with respect to the reference model.

On average, the total amount of CCD applied to each object in order to ensure a good acquisition has been estimated as $7,998 \mathrm{mg} / \mathrm{cm}^{2}$.

Results presented in Fig. 6 show how, after the first hour since the spray was applied, the total number of valid points do not change significantly $(98.44 \%$ of the original points are still present in the 'Pot' case and $98.41 \%$ in the 'Flat glass' case), whilst average acquisition 
deviations are always very close to $0(0.0526 \mathrm{~mm}$ for the 'Pot' and $0.0052 \mathrm{~mm}$ for the 'Flat glass'). During the next four days, the average error does not change significantly, whilst the number of valid measurements gradually decreases, remaining present $77.79 \%$ of the original points for the 'Pot' and $59.44 \%$ for the 'Flat glass' at the end of the experiment.

It can be noticed how sublimation speed changes considerably from one case to the other: in the case of the 'Flat glass', since the air exchange over a big flat surface is greater than in an irregular object as the 'Pot', the evaporation process gets considerably accelerated. However, both objects were perfectly scanned during the first 75 minutes after the application of the spray which, from our experience, provides plenty of time to perform the acquisition.
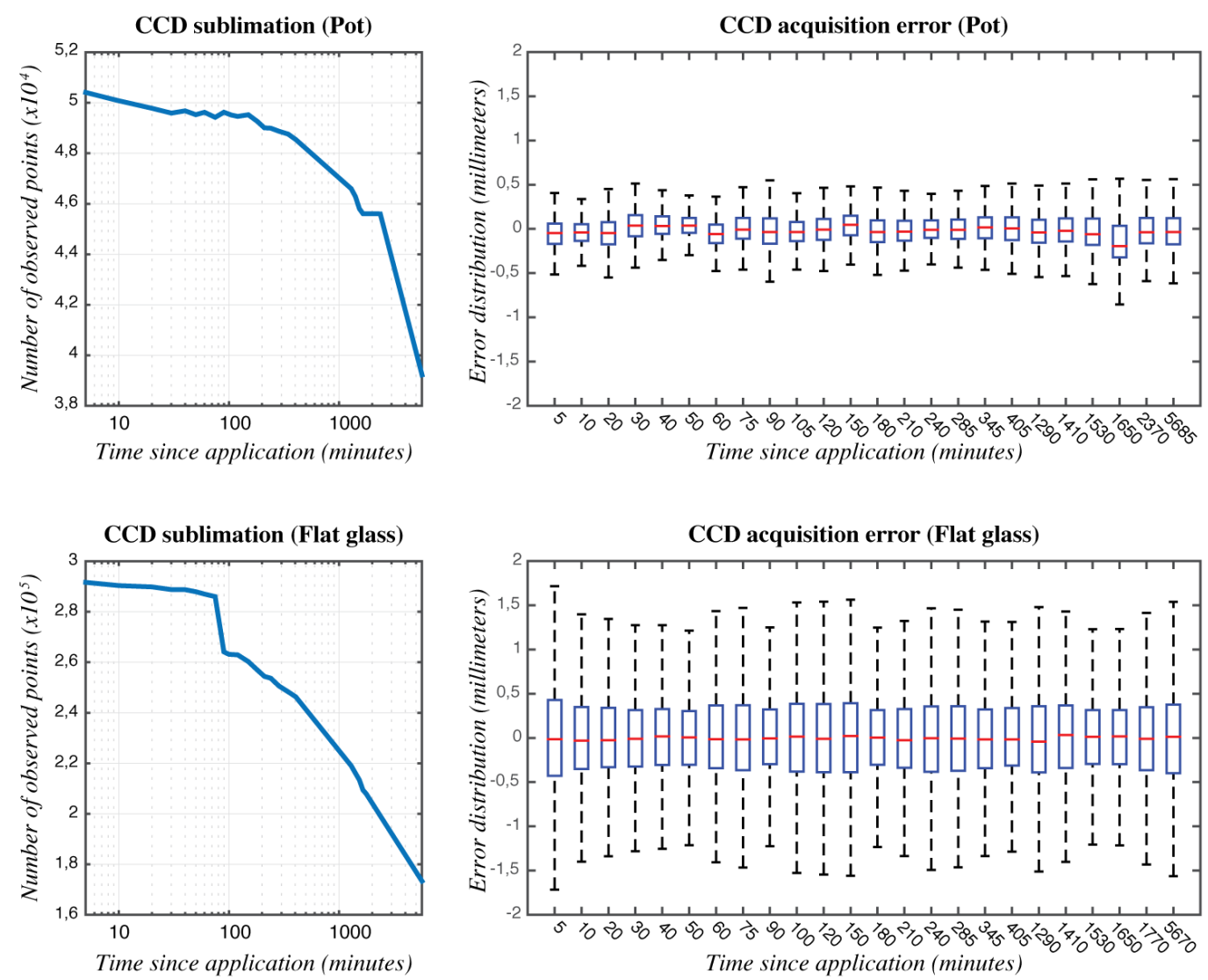

Figure 6: Evaluation of the sublimation speed of CCD. Left charts show the number of valid measures over time with respect to the reference model (acquired immediately after applying the spray), whilst right charts show the error distribution over time.

\section{RESULTS}

According to all previous experiments, the advantages arising from cyclododecane's chemical stability and the fact that it sublimes at ambient temperature make it a perfect candidate for the acquisition of reflective/refractive archaeological artifacts, even taking into account that its particle size is bigger. Also, it is interesting considering that it is a relatively cheap product (cheaper than whitening sprays), and that its toxicity is very low.

Fig. 7 shows the results achieved when scanning a set of glass artifacts using and not using CCD as a whitening spray, whilst Fig. 8 shows one of the artifacts sprayed with cyclododecane for its acquisition, and the same artifact after the white film has completely sublimated, without rubbing or using solvents to remove it. 


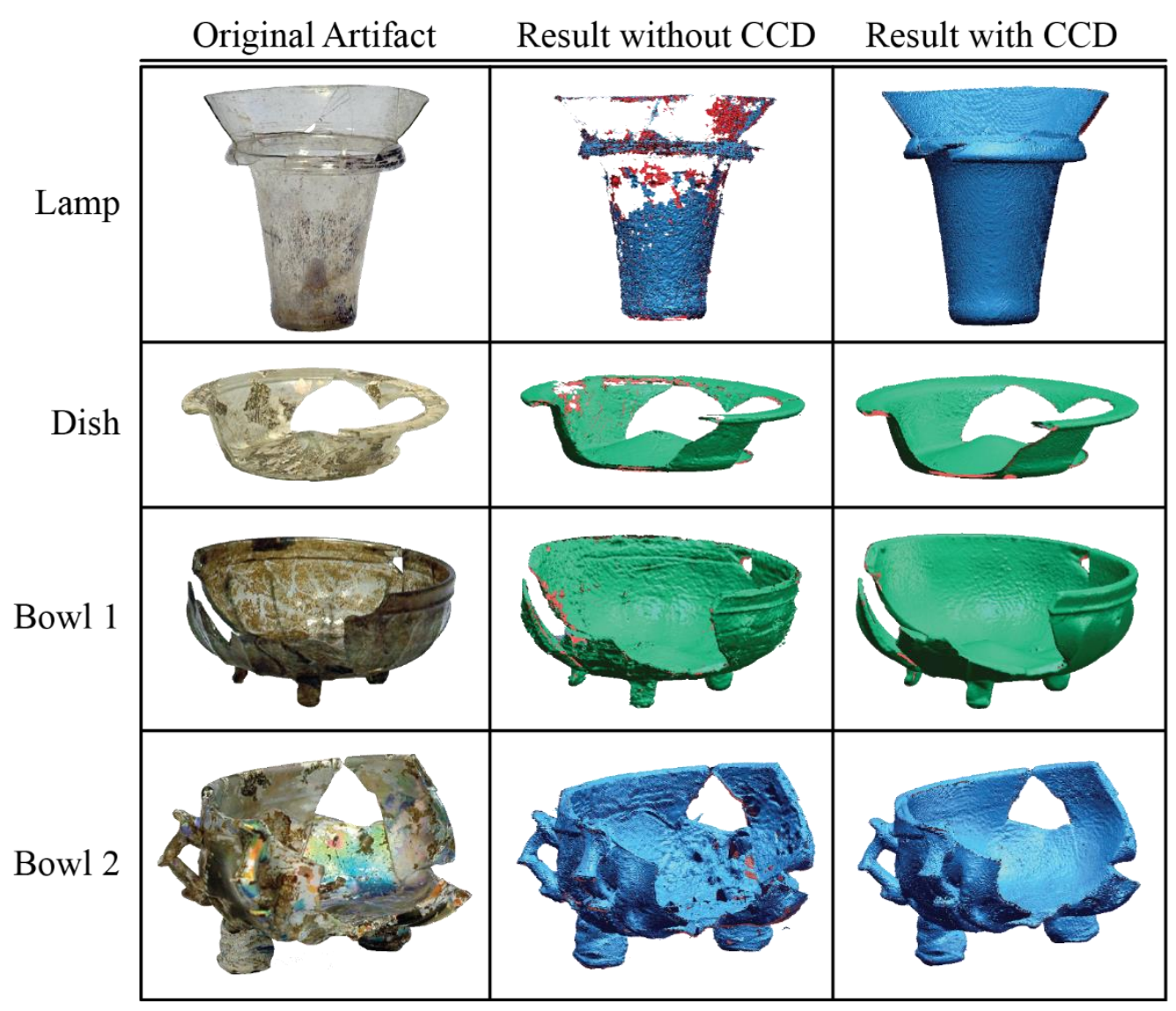

Figure 7: Results achieved using and not using CCD as a whitening spray.

From Fig. 7 it can be appreciated how, for the topmost two artifacts ("Lamp" and "Dish"), transparent areas are not visible to the scanner when CCD is not used and, consequently, the resulting model presents lots of holes. Also, in the "Lamp" model and in "Bowl 1" and "Bowl 2 ", the areas opaque enough to be acquired present lot of noise, due to the reflections/refractions that happen when the laser beam hits the surface. Notice how the use of cyclododecane allows capturing the whole model and reduces considerably the noise introduced by this phenomenon.
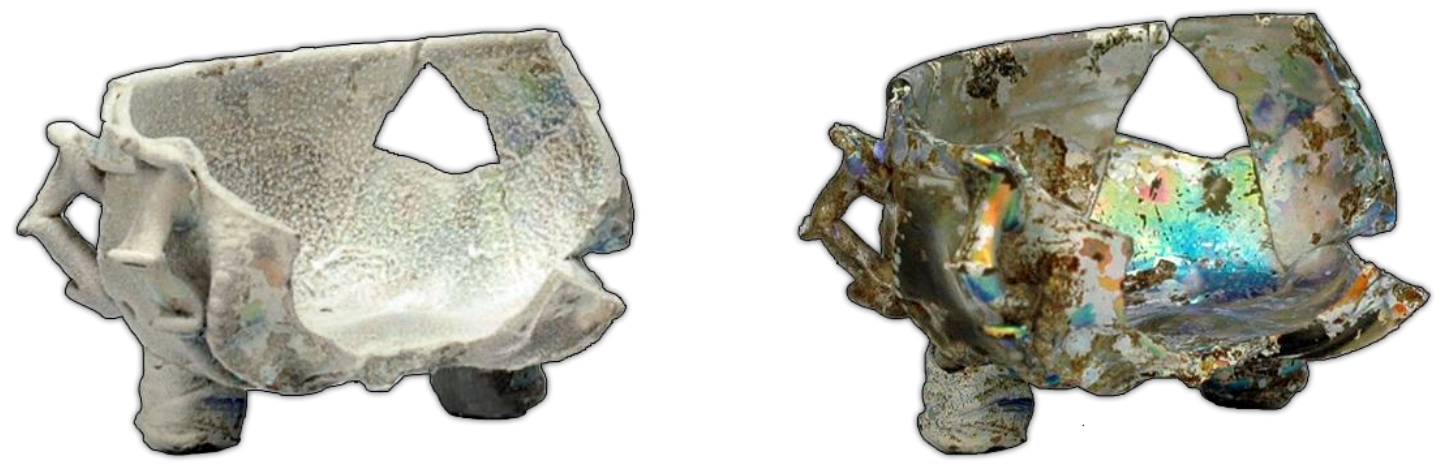

Figure 8: The artifact "Bowl 2" with cyclododecane applied (left), and after the (un-accelerated) sublimation process (right). Notice how no trace of the spray remains on the surface of the object. The sublimation process took 36 hours after acquisition. 


\section{CONCLUSIONS}

This paper has presented an alternative application for a widely used material in heritage conservation and restoration: cyclododecane. Applied as a spray, CCD creates a thin, white opaque film on the surface of artifacts that allows acquiring them using triangulation based 3D laser scanners.

Thanks to its chemical stability and to the fact that it sublimes at room temperature leaving no residuals, CCD is a perfect candidate to solve reflection/refraction issues during the scanning process.

A set of experiments has been presented, proving that the thin layer created on the surface of fragments do not interfere with the scanner accuracy thanks to the reduced particle size.

\section{ACKNOWLEDGEMENTS}

This work is supported by the "Programa de Ayudas de Investigación y Desarrollo (PAID)" of the Universitat Politècnica de València and the "Plan Nacional de I+D+i 2008-2011" from the Ministerio de Economía y Competitividad of Spain, Projects ID: HAR2012-38391-C02-01 and HAR2012-38391-C02-02.

Authors would also like to acknowledge the collaboration to the "Museo de Cerámica de Manises" and especially to its director Mrs. Sara Blanes Ibáñez.

\section{REFERENCES}

[1] G. Salemi, V. Achilli, G. Boatto, 3D Virtual modeling of a gothic stained-glass panel, in: Proceedings published by the International Society of Photogrammetry and Remote Sensing (ISPRS), XXI ISPRS Congress, Volume XXXVII, Part B5, Beijing, July 3-11 (2008) 297-302.

[2] F. Stanco, D. Tanasi, G. Gallo, Virtual restoration of fragmented glass plate photographs of archaeological repertories, Virtual archaeology review (VAR) 3, Volume 2 April (2011) 141144.

[3] I. Ihrke, K.N. Kutulakos, H.P.A. Lensch, M. Magnor, W. Heidrich, Transparent and specular object reconstruction, Computer graphics forum 29 number 8 (2010) 2400-2426. DOI: 10.1111/j.1467-8659.2010.01753.x

[4] F. Mériaudeau, R. Rantoson, D. Fofi, C. Stolz, Review and comparison of non-conventional imaging systems for three-dimensional digitization of transparent objects, Journal of Electronic Imaging 21, number 2 May (2012) 1-6. DOI: 10.1117/1.JEI.21.2.021105

[5] R. Rantoson, C. Stolz, D. Fofi, F. Mériaudeau, Non contact 3D measurement scheme for transparent objects using UV structured light, in: 20th International Conference on Pattern Recognition, Published by IEEE computer society, Istanbul, Turkey, Agost 23-26 (2010) 16461649.

[6] G. Eren, O. Aubreton, F. Meriaudeau, L.A. Sanchez Secades, D. Fofi, A. Teoman Naskli, F. Truchelet, A. Ercil, A 3D scanner for transparent glass, Image Analysis and Processing ICIAP, Lecture Notes in Computer Science Volume 5716 (2009) 519-527.

[7] T. Chen, H.P.A. Lensch, C. Fuchs, H.P. Seidel, Polarization and phase-shifting for 3d scanning of translucent objects, In Proceedings of IEEE Computer Society Conference on Computer Vision and Pattern Recognition (CVPR), Minneapolis MN (2007) 1-8. 
[8] M.B. Hullin, M. Fuchs, I. Ihrke, H.P. Seidel, H.P.A. Lensch, fluorescent Immersion Range Scanning, ACM Transactions on Graphics 27, Issue 3, Article 87 (August 2008). DOI $10.1145 / 1360612.1360686$

[9] S.K. Yeung, T.P. Wu, CH.K. Tang, T.F. Chan, S. Osher, Adequate reconstruction of transparent objects on a shoestring budget, CVPR, IEEE (2011) 2513-2520.

[10] W. Matusik, H. Pfister, R. Ziegler, A. Ngan, L. McMillan, Acquisition and rendering of transparent and refractive object, Eurographics Workshop on Rendering (2002).

[11] H.M. Hangleiter, Elisabeth Jägers, Erthard Jägers, Flüchtige Bindemittel, Zeitschrift für kunst-technologie und konserviertung, 9 (1995) 385-392.

[12] V. Muros, J. Hirx. The use of cyclododecane as a temporary barrier for water-sensitive ink on archaeological ceramics during desalination, JAIC, 43, 75-89.

[13] A. Jeberien, Cyclododecan fürarcháologische Funde? Bergumg stark fragmentierter keramik der Hallstattzeit, Restauro 108 (2002) 509-511.

[14] M. Berthet, E. Fraile-Ugalde, Cyclododecane as temporary fixative in paper conservation, Paper Conservation News 111, 2004, 13.

[15] M. Brown, A. Davidson, The use of cyclododecane to protect delicate fossils during transportation, Journal of Vertebrate Paleontology 30:1 (2010) 300-303. DOI:

$10.1080 / 0274630903413123$.

[16] S. Rowe, C. Rozeik, The uses of cyclododecane inconservation, International Institute for Conservation of Historic and Artistic Works IIC, reviews in conservation 9 (2008) 17-31.

[17] D.C. Cleere, Cyclododecane reinvestigated: and experimental study on using cyclododecane to secure unstable ceramic surfaces prior to transportation, Conservation News 94 (2005) 26-28.

[18] Krzystof Skabek and Przemyslam Kowalski, Building the models of cultural heritage objects using multiple 3D scanners, Theoretical and Applied Informatics vol. 21 (2009) no.2 115-129.

[19] G. Celani, L. Cancherini, A. Jardini, M. Oliveira, J. V. Lopes da Silva, R. Archer, V. Piccoli, 3D digitation of museum sculptures for model-making purposes: difficulties and possible solutions, Anais do VRAP (2009).

[20] C. Anselmi, F. Presciutti, B. Doherty, B.G. Brunetti, A. Sgamellotti, C. Miliani, The study of cyclododecane as a temporary coating for marble by NMR profilometry and FTIR reflectance spectroscopies, Applied Physics A 104 (2011) 401-406.

[21] R. Stein, J. Kimmel, M. Marincola, F. Klemm, Observations on cyclododecane as a temporary consolidant for stone, JAIC 39 (2000) 355-369.

[22] S. Caspi, E. Kaplan, Dilemmas in transporting unstable ceramics: a look at cyclododecane, Smithsonian National Museum of the American Indian (2001).

[23] C. Watters, Cyclododecane: a closer look at practical issues, AAS: GXVI (2007) 195-204.

[24] M. Neurne, M.O. Hubert, "Consolidation et protection temporaire: un exemple de traitement des matériaux vitreux à l'aide de cyclododécane", CoRé: Conservation et Restauration du Patrimonie Culturel 10, 2001, 18-21. 